\title{
Editorial
}

\section{Pat Croskerry}

\section{Sapere aude in the diagnostic process}

https://doi.org/10.1515/dx-2020-0079

With sapere aude in his First Book of Letters (20 BCE), the Roman poet Horace exhorts decision makers to have the courage and understanding to use reason and intellect in their pursuit of knowledge and truth. It became Kant's motto for the entire Age of Enlightenment, [1] which began around the turn of the 18th century. It's main driving force was the attainment of rationality. While we have since reaped the benefits of centuries of individual liberties and enjoyed relative freedom from dogma, superstition, and other nonscientific constraints, Kant [1], Habermas [2] and others have argued, nevertheless, that the enlightenment itself remains an unfinished business. Modern decision making generally, and clinical decision making in particular still fall short of the ideal, and we continue to fail in significant ways. In a much-cited paper, the diagnostic failure rate is put at about $10-15 \%$ [3]. Most of us would not cross the road against those odds.

Optimal diagnosis depends upon optimal decision making which, in turn, depends upon optimal rationality. In fact, we can refine this further and say that clinical prediction is the main challenge. Diagnosis is about prediction-how accurately can a clinician predict the identity of one (or more) of about 12,000 diseases that may underly the patient's limited repertoire of symptoms and signs? Inevitably, prediction is made more difficult by the number of diagnoses far outweighing the clues that the human body may manifest. In Enlightenment now. The case for reason, science, humanism and progress, Stephen Pinker argues that 'the acid test of empirical rationality is prediction' [4]. Rationality, itself may take a variety of forms, but if we adopt the normative view that 'Our actions and beliefs are justifiable [or reasonable/rational] as a function of the trustworthiness of the evidence, and the extent to which we believe that evidence is determined by credible processes' [5] (such as evidence-based medicine), then the

Pat Croskerry, Dalhousie University, Emergency Medicine, Halifax Infirmary Suite 355-1796 Summer Street, Halifax, Nova Scotia, B3H 4R2, Canada, E-mail: pgcxkerry@gmail.com question is: what can we do to improve the predictive power of our decision making to increase the likelihood of a correct diagnosis and reduce the morbidity and mortality of diagnostic failure?

A necessary first step is an appreciation of the complexity of the diagnostic process. It is a large coin with two different but critically important sides. On the one side, medical knowledge in all its breadth and depth is a mandatory foundation, without which attempts at diagnosis would be fruitless. Such knowledge is declarative - the nuts and bolts of medicine from the Krebs Cycle to the contents of Harrison's Principles of Internal Medicine. However powerful and deep the intellect, and however brilliant the reasoning, the process will inexorably fail without sufficient declarative knowledge. Upon this base operate upwards of over 40 factors clustering into six major groups: individual characteristics of the decision maker, individual intellectual and cognitive styles, ambient and homeostatic factors, factors in the work environment including team factors, characteristics of the medical condition, and factors associated with the patient. Additional factors, such as health care systems, culture, politics and others also exert important influences [6]. All contribute in quantitative and qualitative ways to the context in which reasoning and decision making occur, so it is important to be aware of and anticipate contextual factors [7]. Importantly, medical declarative knowledge, as it is currently acquired in Western Medicine training, does not appear to be a significant limiting factor in the diagnostic process [8-11]. Mostly, it is how we think that gets us into trouble rather than what we don't know.

On the obverse of the coin is the calibre of the physician's decision making and procedural knowledge - how doctors think. Many of the 40 factors described above include aspects of the ways in which clinicians make decisions. No two clinicians are alike in this regard, in fact, they are often distinctly unalike. Gender, age, experience, personality, decision making styles, religion, culture and other factors significantly influence competence in how to think and calibration of decision-making [12] Other characteristics of the system in which decisions are made are also important, including context which establishes the situational topography upon which many decisions are made in medicine [7]. The collection of papers in this issue of Diagnosis attempt to throw further light on the role of context. 


\section{The challenge of research into context}

Typically, the process of investigation of any phenomenon begins with observation and description of the dependent variable in its natural setting. Various models may then be generated to provide an overall understanding of the main factors that appear to be relevant and how they interact with each other. Several models of human decision making have emerged over the last 50 years, a period in which cognitive psychology has flourished. A leading contender emerged in the form of dual process theory (DPT), [13-15] (though an excellent earlier description of DPT can be found in Thomas Paine's Age of Reason [1794]) [15]. Which now appears well-established in the medical literature [16-20]. Not only does it delineate the two main processes (intuitive and rational) by which decisions get made, but also allows some insight into how intuition is progressively accrued and refined by experience. Intuition is a broad term in decision making, and has different meanings for different people [21]. Thomas Paine recognised it as a valuable form of understanding [15]. Myers defines it as "our capacity for direct knowledge, for immediate insight without observation or reason." [22] Stanovich, in his book, describes four sub-processes through which intuition might be established: (i) hard-wired, genetically transmitted, naturally selected origins, (ii) those associated with emotions, inherited or acquired, (iii) those that have been acquired through explicit learning and practice, and (iv) those acquired through implicit learning [23]. For the rational process, things appear a little more straightforward, although the term may have specialised meanings in different domains, and also within medicine itself [5]. Overall, despite these variations in use and definition, DPT has provided a useful platform for investigating various aspects of decision making.

Often, what typically follows model development is a strategy of reductionism in which independent variables are identified and their individual impact on the dependent variable assessed. This is usually done in an experimental setting in the laboratory where they are experimentally isolated in order to exclude their influence. However, there are several problems in applying this process to the study of diagnosis. As noted, the dependent variable (diagnosis) is influenced by multiple independent variables (the 40+ described above). Firstly, there are ethical challenges in studying the problem in its natural habitat where randomised, double-blind, prospective studies are nigh impossible. Secondly, studying diagnosis in the laboratory may be severely compromised by taking the process out of its natural setting. The problem was summarised very well by Gruppen and Frohna: “...too often, studies of clinical reasoning seem to take place in a vacuum. A case or scenario is presented to subjects, usually in written form, stripped of any 'irrelevant' noise. The traditional methodology of providing clinical cases that are decontextualized and 'clean' may not be a particularly valid means of assessing the full range of processes and behaviors present in clinical reasoning in natural settings." [24] The main issue here is decontextualizing which may draw the ecological validity of the study into question. Similarly, Wears and Nemeth [25] proposed abandoning laboratory studies in favour of 'real world' studies that focus on context and on the intuitive processing that experts use. Such post hoc analyses should be done to reconstruct the clinician's experience at the time of the event, perhaps by interviewing the clinician, or by promoting self-awareness and introspection. Thus, the common method of studying diagnosis using computer screens that display clinical vignettes may be no more than pale imitations of what actually happens in the clinical situation. Yet, a review of studies of cognitive biases and heuristics in medicine found that $77 \%$ of 213 studies reviewed were based on hypothetical vignettes [26]. While an advantage of this approach is that studies can be done with relative ease, and the appearance of experimental respectability may hold some appeal for editors and their reviewers, their context is almost non-existent and their ecological validity may be seriously questioned [27].

There are several ways in which we can study the diagnostic process without falling into this ecological trap [28]. One is by looking at diagnostic failure using standardised or simulated patients (SP). The SP presents to a clinic unannounced and is seen and assessed 'blind' by the clinician. In one study, the diagnostic failure rate using this methodology was estimated at $13 \%$, [29] with the advantage that much of the medical context was preserved. A major problem with the procedure, however, is that the SP is trained to simulate prototypical manifestations of a particular disease, an example of representative bias [30]. Prototype and the representative bias are characteristic of an anticipated context. Inevitably, atypical presentations are not investigated using this method. This is an important constraint for some illnesses e.g. atypical presentations of acute myocardial infarction (without chest pain) occur about $1 / 3$ of the time [31]. If experimenters programmed SPs for atypical presentations of illness, this methodology would achieve an even better level of ecological validity. Another method is through the use of in vivo studies in which real clinical cases are rigorously subjected to cognitive root cause analysis, with particular attention paid to prevailing context [11]. This requires that those 
conducting the analysis have a sound understanding of the multiple sources of error in clinical situations, and especially of cognitive failings.

Another issue for the diagnostic process that illustrates vulnerability to the ecological problem is Artificial Intelligence (AI). While AI can employ machine learning (ML) techniques for such complex problems as diagnosis by making predictions from very large amounts of patient data by learning ML's own associations, [32] the data is typically inputted without context. Part of the problem is that while implicit awareness of context may often be factored into clinical decision making in real life it may not be so easily articulated for AI systems. A particular problem is 'distribution shift' where ML systems may fail to recognise that a significant change in context has occurred. Challen et al. define distribution shift as: 'A mismatch between the data or environment the system is trained on and that used in operation, due to bias in the training set, change over time, or use of the system in a different population, resulting in an erroneous 'out of sample' prediction' [33]. A pandemic is a case in point, where new data and environmental changes may appear overnight.

\section{An abrupt change of context}

A significant current example of the impact of context is the Covid-19 pandemic, which has abruptly changed the clinical practice of medicine worldwide. It has been the ultimate context-changer. Potentially, every patient seen may have the disease, with the decision makers themselves being no exception. The signs and symptoms of presentation do not provide a pathognomonic pattern that may be readily recognised, and consequently other diseases may easily be mimicked. We can expect that several common cognitive biases normally associated with the diagnostic process will be in force, principally availability, as well as others (anchoring, framing, ascertainment bias, unpacking failure, search satisficing, diagnosis momentum, groupthink, and confirmation bias). Communication, a key aspect of the diagnostic process, is significantly changed, through provider's donning personal protective equipment, especially facial masks, thereby attenuating significant aspects of body language. Ambient factors in the workplace will alter context: increased workload, cognitive load, stress levels and other factors known to influence the calibration of decision making are all changed. Emotional issues for providers are changed, not the least being increased fear levels for themselves and their families should they contract the disease, but also the toll of compassion fatigue through recurrent episodes of dysphoria associated with witnessing death.

\section{What does sapere aude ask of us?}

We need to accept that the process of diagnosis, although often straightforward and not requiring exceptional effort, can be tricky and deceptive at times, and will require reason and intellect in the attainment of diagnostic accuracy. An important part of our intellect is an awareness of the prevalent biases that may impact our decision making, awareness of our failures to reason logically, a capacity for mindfulness, and a commitment to paying attention [34]. Often, to be mindful requires very little extra effort yet may achieve significant results. Being mindful of the impact of context, of the constant intrusion of bias, of the ambient conditions in which we work, and of our own homeostasis and well-being allows us to keep a check on our decision making. In particular, we need to be aware that what you see is all there is (WYSIATI) [35] may be a harbinger of premature closure. Overall, we need to understand the complexity of a process that we often take for granted, and be more skeptical when things appear obvious. The novelist Lawrence Durrell saw this as our greatest challenge: 'The greatest delicacy of judgement, the greatest refinement of intention was to replace the brutish automatism with which most of us exist, stuck like prehistoric animals in the sludge of our non-awareness' [36]. It would seem that dealing with our 'brutish automatism' and un-sticking ourselves from the 'sludge of non-awareness' cannot be accomplished without at least some degree of mindfulness. Importantly, this has recently received some emphasis in an AHRQ report into patient safety: 'Although there are a limited number of studies, the literature suggests that training on metacognitive skills may improve diagnostic accuracy, particularly as clinical experience increases' [37]. Finally, to re-visit Kant: 'If it is now asked whether we at present live in an enlightened age, the answer is: No, but we do live in an age of enlightenment' [1]. The greater detail with which we now understand the diagnostic process signifies not that medicine currently exists in a fully enlightened age (that may not be attainable for some time), but at least it is within an age of enlightenment, where we can steadily improve it. 
Research funding: None declared.

Author contributions: The author has accepted responsibility for the entire content of this manuscript and approved its submission.

Competing interests: The author states no conflict of interest.

\section{References}

1. Kant I. An answer to the question: "what is enlightenment?" Konigsberg in Prussia. 30th September, 1784. https://www. marxists.org/reference/subject/ethics/kant/enlightenment.htm.

2. Habermas J. The philosophical discourse of modernity, twelve lectures. Trans. Frederick Lawrence. Cambridge, MA: Massachusetts Institute of Technology; 1987.

3. Berner ES, Graber ML. Overconfidence as a cause of diagnostic error in medicine. Am J Med 2008;121(5 Suppl):S2-23.

4. Pinker S. Enlightenment now. The case for reason, science, humanism and progress. New York, NY: Viking; 2018.

5. Djulbegovic B, Elqayam S, Dale W, Coppola AM. Rational decision making in medicine: implications for overuse and underuse. J Eval Clin Pract 2018;24:655-65.

6. Croskerry P. Adaptive expertise in medical decision making. Med Teach 2018;40:803-8.

7. Croskerry P. Context is everything or how could I have been that stupid? Healthcare quarterly; special issue: understanding decision-making in healthcare and the law 2009;12:167-73.

8. Graber ML, Franklin N, Gordon R. Diagnostic error in internal medicine. Arch Intern Med 2005;165:1493-9.

9. Gruver R, Freis E. A study of diagnostic errors. Ann Intern Med 1957;47:108-20.

10. Kiesewetter J, Ebersbach R, Tsalas N, Holzer M, Schmidmaier R, Fischer MR. Knowledge is not enough to solve the problems - the role of diagnostic knowledge in clinical reasoning activities. BMC Med Educ 2016;16:303.

11. Croskerry P. The cognitive autopsy. A root cause analysis of medical decision making. New York, NY: Oxford University Press; 2020.

12. Croskerry $P$. Individual variability in clinical decision making and diagnosis. In: Croskerry P, Cosby K, Graber M, Singh H, editors. Diagnosis: interpreting the shadows. Boca Raton, FL: CRC Taylor and Francis Group; 2017.

13. Schneider W, Shiffrin RM. Controlled and automatic human information processing: 1 . Detection, search, and attention. Psychol Rev 1977;84:1-66.

14. Evans, J. Heuristic and analytic processes in reasoning. $\mathrm{Br}$ J Psychol 1984;75:451-68.

15. Paine T. The age of reason. San Bernardino, CA: Minerva Publishing; 2018:35 p.

16. Dawson NV. Physician judgment in clinical settings: methodological influences and cognitive performance. Clin Chem 1993;39:1468-80.

17. Croskerry P. The theory and practice of clinical decision making. Can J Anesthesiol 2005;52:R1-8.

18. Croskerry P. A universal model for diagnostic reasoning. Acad Med 2009;84:1022-8.
19. Brush JE. The science of the art of medicine; 2015. https:// itunes.apple.com/us/book/science-art-medicine/ id 643948555 ? $\mathrm{mt}=13$.

20. Croskerry P, Graber M, Cosby K, Singh H. Diagnosis: interpreting the shadows. Boca Raton, FL: CRC Taylor and Francis Group; 2017.

21. Croskerry P. The rational diagnostician. In: Croskerry P, Cosby K, Graber M, Singh H, editors. Diagnosis: interpreting the shadows. Boca Raton, FL: CRC Taylor and Francis Group; 2017.

22. Myers DG. Intuition: its powers and perils. New Haven, CT: Yale University Press; 2002.

23. Stanovich KE. Rationality and the reflective mind. New York: Oxford University Press; 2011:19-22 p.

24. Gruppen LD, Frohna AZ. Clinical reasoning. In: Norman GR, van der Vleuten CP, Newble DI, editors. International handbook of research in medical education. Boston, MA: Kluwer Academic; 2002:205-30 pp.

25. Wears RL, Nemeth CP. Replacing hindsight with insight: toward better understanding of diagnostic failures. Annals Emerg Med 2007;49:206-9.

26. Blumenthal-Barby JS, Krieger H. Cognitive biases and heuristics in medical decision making: a critical review using a systematic search strategy. Med Decis Making 2015;35:539-57.

27. Croskerry P, Petrie DA, Reilly JB, Tait G, Deciding about fast and slow decisions. Acad Med 2014;89:197-200.

28. Graber ML. The incidence of diagnostic error in medicine. BMJ Qual Saf 2013;22:ii21-7.

29. Peabody JW, Luck J, Jain S, Bertenthal D, Glassman P. Assessing the accuracy of administrative data in health information systems. Med Care 2004;42:1066-72.

30. Kostopoulou O, Oudhoff J, Nath R, Delaney BC, Munro CW, Harries $\mathrm{C}$, et al. Predictors of diagnostic accuracy and safe management in difficult diagnostic problems in family medicine. Med Decis Making 2008;28:668-80.

31. Canto JG, Shlipak MG, Rogers WJ, Malmgren JA, Frederick PD, Lambrew CT, et al. Prevalence, clinical characteristics, and mortality among patients with myocardial infarction presenting without chest pain. J Am Med Assoc 2000;283:3223-9.

32. Liang H, Tsui BY, Ni H, Valentim CCS, Baxter SL. Guangjian L, et al. Evaluation and accurate diagnoses of pediatric diseases using artificial intelligence. Nat Med 2019;25:433-8.

33. Challen R, Denny J, Pitt M, Gompels L, Edwards T, TsanevaAtanasova K. Artificial intelligence, bias and clinical safety. BMJ Qual Saf 2019;28:231-7.

34. Epstein R. Attending: medicine, mindfulness, and humanity. New York, NY: Scribner; 2017.

35. Kahneman D. Thinking fast and slow. New York: Farrar, Straus and Giroux; 2011.

36. Durrell L. A smile in the mind's eye: an adventure into zen philosophy. London: Open Road Media; 2012.

37. Hall KK, Schiff G, Witgert K. Patient safety practice: education and training. In: Hall KK, Shoemaker-Hunt S, Hoffman L, Richard S, Gall E, Schoyer E, Costar D, et al., editors. Making healthcare safer III: a critical analysis of existing and emerging patient safety practices. AHRQ Publication No. 20-0029-EF. Rockville, MD: Agency for Healthcare Research and Quality; March 2020. 